Itinéraires Itinéraires

Littérature, textes, cultures

2012-2 | 2012

Intime et politique

\title{
Hélène Hoppenot, Journal 1918-1933
}

Édition annotée, établie et préfacée par Marie-France Mousli, Paris, Éditions Claire Paulhan, 2012, 639 pages.

Hélène Gestern

\section{OpenEdition}

12 Journals

Édition électronique

URL : http://journals.openedition.org/itineraires/1208

DOI : $10.4000 /$ itineraires. 1208

ISSN : 2427-920X

Éditeur

Pléiade

Édition imprimée

Date de publication : 1 novembre 2012

Pagination : 168-172

ISBN : 978-2-336-00027-5

ISSN : $2100-1340$

Référence électronique

Hélène Gestern, « Hélène Hoppenot, Journal 1918-1933 », Itinéraires [En ligne], 2012-2 | 2012, mis en ligne le 01 novembre 2012, consulté le 22 septembre 2020. URL : http://journals.openedition.org/ itineraires/1208; DOI : https://doi.org/10.4000/itineraires.1208

Ce document a été généré automatiquement le 22 septembre 2020.

\section{(c) (ஒ) $\odot$}

Itinéraires est mis à disposition selon les termes de la licence Creative Commons Attribution - Pas d'Utilisation Commerciale - Pas de Modification 4.0 International. 


\section{Hélène Hoppenot, Journal 1918-1933}

Édition annotée, établie et préfacée par Marie-France Mousli, Paris, Éditions Claire Paulhan, 2012, 639 pages.

\section{Hélène Gestern}

\section{RÉFÉRENCE}

Hélène Hoppenot, Journal 1918-1933, édition annotée, établie et préfacée par Marie-

France Mousli, Paris, Éditions Claire Paulhan, 2012, 639 pages.

C'est encore un petit bijou que nous livrent les éditions Claire Paulhan avec la première édition du Journal 1918-1933 d'Hélène Hoppenot. Cette parution était attendue depuis longtemps par ceux qui avaient pu lire des extraits du texte, déposé du vivant de son auteur à la Bibliothèque Jacques-Doucet ; elle saura passionner tous ceux qu'intéressent les journaux personnels, mais aussi la vie littéraire de la première moitié $\mathrm{du} \mathrm{xx}^{\mathrm{e}}$ siècle, l'histoire mondiale de l'entre-deux-guerres et les récits de voyage. Ces six cents pages, dont Marie-France Mousli offre une édition scrupuleuse, sont enrichies de nombreuses photographies, de documents, de cartes, de citations croisées de journaux d'Henri Hoppenot et de Claudel ainsi que d'un index, qui ajoutent à leur valeur documentaire intrinsèque déjà grande. Voici le récit d'une expérience de vie peu commune, puisque durant ces quinze années, les Hoppenot connaîtront huit affectations diplomatiques sur deux continents, à une période où l'échiquier politique, partout instable, laisse déjà lire les prémices de la catastrophe à venir. Si la matière du journal est passionnante, le style ne l'est pas moins: bien qu'Hélène Hoppenot ait déploré son absence de qualité littéraire (" des notes écrites au courant de la plume, [...] à la va-comme-je-te-pousse »), on est séduit par son écriture vivace, alerte, précise comme un instantané photographique. 


\section{Une jeune femme pas comme les autres}

2 Hélène Delacour naît en 1894 dans la famille d'un militaire, ce qui lui vaut de connaître une enfance itinérante. Peu tentée par le mariage, bonne pianiste, elle se destine à une carrière de musicienne - elle se produira d'ailleurs, en qualité de chanteuse, dans quelques récitals parisiens. Sa rencontre en 1915 avec Henri Hoppenot, un jeune homme réformé pour handicap qui vient d'entrer au Quai d'Orsay, bouleverse ses projets. Certes, le métier d'épouse de diplomate, car c'est bien une profession à part entière, ennuie Hélène par son côté mondain. Mais il lui offre la possibilité d'assouvir une passion des voyages ("Départ! Voyage! Deux mots aimés.») poussée chez elle à son paroxysme : «Comme j'aimerais faire craquer cette planète trop étroite, sauter sur l'un de ses morceaux et partir à la découverte de l'univers!», écrit-elle en 1927. Les Hoppenot, on le devine, sont un couple peu conformiste : ils se sont mariés civilement et Hélène est résolument athée. La diariste a toujours une moquerie en réserve pour les ecclésiastiques, tels cet ancien prêtre et cette ancienne religieuse, qui se sont, dit-elle, " consolés ensemble des déboires de la religion "; d'un père jésuite ambitieux, elle note, ironique, qu'il «ne perd pas son temps à évoquer le seigneur ». L'athée est aussi féministe: à Téhéran, le confinement réservé aux femmes la navre. On trouve de nombreuses piques, sous sa plume, contre le tchadour et la domination masculine : « En Asie, plus que partout ailleurs, l'homme a conçu la religion, la loi, selon son bon plaisir, la femme étant son jouet et son esclave : ici, il joue au naturel le rôle de dominateur, pour lequel il se croit créé.» Hélène Hoppenot demeure cependant confiante en l'évolution de la condition féminine, qu'elle dépeint en des termes visionnaires pour l'époque: «Un jour, [les femmes] feront leurs lois, deviendront libres de procréer ou sans crainte de l'affreux scandale d'avorter : ce sera leur âge d'or " (2 mars 1923). Ellemême, qui bourlingue dans des contrées instables, travaille aux côtés de son mari, fréquente les milieux littéraires parisiens lors de ses passages en France, fait de longues promenades à cheval avec ses chiens et deviendra une remarquable photographe de l'Asie, est l'archétype d'une femme que l'on dirait aujourd'hui « libérée ».

\section{Un journal personnel}

3 Le journal d'Hélène Hoppenot est plus personnel qu'intime. La diariste, durant les trois premières années, n'y évoque jamais ses états d'âme, et ne décrit que rarement ses relations avec son mari. On devine toutefois qu'elle est liée par une affection profonde (et réciproque) à celui qu'elle ne désigne jamais autrement que par son initiale, « $\mathrm{H}$. » Claudel, frappé par leur complicité, les surnomme d'ailleurs le «casal », le « couple » en portugais. Les indications qu'Hélène donne sur leur vie quotidienne montrent deux êtres en harmonie, unis par leurs passions communes: la curiosité intellectuelle, les voyages, le goût de la découverte, la collection d'objets d'art, l'amour de la peinture (ils aiment Juan Gris), la photo. Le peu de goût d'Hélène pour l'introspection va de pair avec un sens de l'humour aigu, une résistance physique aux aléas des voyages - à l'époque, se rendre à Téhéran en plein hiver n'est pas une mince affaire -, un réel courage (elle refuse de quitter son mari devant la menace de l'arrivée de l'armée russe) et une bonne dose de pragmatisme.

4 Toutefois, la femme a des blessures secrètes : une mère et des beaux-parents qu'elle n'aime pas, et surtout une maternité douloureusement vécue. Elle tombe enceinte en 
1922, cinq ans après son mariage et l'accepte mal: "Je n'ai que le sentiment du désastre ", écrit-elle le 4 janvier 1923. Il est rare d'entendre une femme avouer, comme elle le fait, à quel point une grossesse non désirée peut être perçue comme une catastrophe. Hélène ne se sent pas le moindre instinct maternel, est constamment malade et regrette déjà sa liberté perdue : quinze jours avant la naissance de sa petite fille, elle note : « voici qu'un intrus va venir, plus pressant chaque jour, affirmant sa vie en tourmentant la mienne... Drame qui me révolte ». La grossesse sera physiquement pénible, au point que la jeune femme, qui se trouve laide et morose, utilisera son journal pour s'exhorter à se reprendre. L'accouchement dure trente-quatre heures, et il est manifestement suivi d'une sévère dépression, qui laissera un souvenir de cauchemar. Peut-être est-ce pour cette raison que la petite fille des Hoppenot sera fort peu mentionnée dans le journal, même si l'affection fait son chemin avec le temps : cinq ans plus tard, en 1928, à Damas, Hélène note: «je désire que Violaine, sans s'en apercevoir, prenne l'habitude d'aimer autant les fleurs que les animaux ».

5 Si l'on excepte l'aveu de cette détresse, unique épisode mélancolique dans le journal, l'intériorité se dit surtout dans l'enthousiasme et les accents lyriques, qui surgissent lorsque la diariste évoque pays et paysages. Du Brésil, elle célèbre la «beauté lourde, ténébreuse, impénétrable»; de la Méditerranée, "la couleur de pêche mûre de la lumière ", du Liban, les " cyclamens vanillés, multicolores » et les « anémones blanches, larges, somptueuses » qui réjouissent son œil d'observatrice.

\section{Un regard critique}

6 Si le journal d'Hélène Hoppenot se révèle aussi intéressant, c'est justement parce qu'il témoigne, dans l'espace privé de l'écriture diaristique, d'une vision passionnée, engagée... mais aussi critique, des pays traversés. L'éditrice évoque - dans un souci de précaution tout contemporain - l'absence de « politiquement correct » dans ces pages... on ne peut que s'en féliciter! Bien sûr, Hélène Hoppenot partage quelques-uns des préjugés de son temps, et il lui arrive d'avoir la dent dure avec certains peuples qu'elle n'aime pas. Au Chili, où elle souffre de dépression (elle $\mathrm{y}$ fonde avec de jeunes francophiles le «Club du Cafard»), elle prend les habitants en grippe et parle du « dédain qu'[elle] éprouve pour ces Sud-Américains ignorants, inéduqués, stupides » auxquels elle préfère... son chien. Elle moque les marchands d'art juifs et persans qui tentent de l'appâter, ou encore philosophe sur l'honnêteté toute relative de son personnel de maison à Téhéran. Et surtout, elle nourrit de lourdes préventions contre les Allemands : «Malgré toute ma volonté d'oublier une guerre atroce, pour essayer de comprendre les Allemands et peut-être de les aimer [...], mon instinct me crie que toute amitié, entre nous, est impossible » (9 octobre 1926). Mais on ne saurait soupçonner de racisme cette femme intelligente, qui noue des amitiés avec les autochtones dans tous les pays et tente toujours d'en comprendre la culture. Elle aime les Brésiliens « que leur sang noir ou indien rend si sympathiques » et s'amuse d'une relation amicale matoise entretenue avec un marchand persan. Toute femme de diplomate soit-elle, elle laisse échapper son exaspération contre le prosélytisme (« Pourquoi voulez-vous assimiler [les Orientaux] ?, dis-je, à bout de patience. Ne peut-on pas laisser ces peuples tranquilles, eux, et leurs traditions?») et les certitudes colonialistes la laissent sceptique. L'injustice et l'arrogance de classe lui déplaisent; en Perse, elle se refuse à battre ses domestiques 
comme c'est l'usage, renvoie ses gardes cosaques trop brutaux, et ne manifeste guère d'indulgence pour les consuls et les ministres qui humilient leurs subordonnés.

7 Mais surtout, Hélène seconde son mari dans ses nombreuses obligations diplomatiques, ce qui lui donne l'occasion de dresser des portraits piquants et lucides des microcosmes d'expatriés qu'elle côtoie. Celle qui deviendra en Asie une excellente photographe possède un véritable talent de caricaturiste, l'art de débusquer le trait saillant, d'épingler les petits ridicules dans les cercles où elle gravite. Ses portraits de Claudel, ministre plénipotentiaire au Brésil (Henri Hoppenot y sera son secrétaire d'ambassade) sont désopilants : elle le trouve caractériel, autoritaire... et fantasque, ce dernier trait rachetant pour elle tous les autres. Voici ce que donne, sous sa plume (caustique), la description d'une expédition pédestre de deux jours : «leur capacité de sommeil fait rugir Claudel qui ne peut admettre que les autres se reposent dès qu'il est, lui, réveillé. Sa vie au Brésil est si chaste qu'il semble avoir oublié que les autres peuvent goûter les divertissements de la nuit et en ressentir quelque fatigue le matin» (27 juillet 1918). Elle se moque de la femme de l'ambassadeur de France en Allemagne, qu'elle soupçonne de "dormir avec des bigoudis", de celle de l'ambassadeur d'Angleterre, avec son "visage de jument anémique ", d'un prince persan qui « ressemble à un scarabée tombé sur le dos ». En même temps, celle que l'on pourrait comparer, en termes de verve stylistique, à une Mme de Sévigné dépeignant le corps diplomatique, est aussi pleine d'indulgence et de bonté, quand elle évoque Supervielle (homme «doux, gauche et mélancolique »), son amie Satie ("son humour, sa drôlerie, son regard pétillant de malice »), les Milhaud qu'elle adore ou un jeune professeur de français avec qui son époux et elle font la paix suite à un petit différend littéraire: «Il eût été dommage, écrit Hélène, de contrister cet homme probe et sincère ».

Celle qui se disait «fascinée par l'expérience nouvelle, l'instabilité des jours, les rencontres imprévues et les imprévisibles » a su en livrer, dans ce journal qu'elle a tenu toute sa vie, jusqu'aux années 1980, la quintessence: une relation pertinente, analytique, drôle et vivante, servie par une plume pleine de finesse et de dynamisme. Même s'il ne lui ressemble en rien, le journal d'Hélène Hoppenot présente un point commun avec celui de Catherine Pozzi : il se dévore comme un roman. 